Willgerodt: Die Halogenüberträger ete. 391

\title{
Die Halogenüberträger in den natürlichen Gruppen und den Perioden der Elemente;
}

\author{
von
}

\section{Willgerodt.}

Um ein übersichtliches Bild von der Stellung der Halogenüberträger im periodischen und dem dadurch geschaffenen natürlichen Systeme der Elemente zu erhalten, mussten noch eine ganze Reihe von Versuchen neuausgeführt, zum Theil auch wiederholt werden. Nur allein dadurch, dass mir Hr. Bergrath Prof. Dr. Clemens Winkler mit der grössesten Bereitwilligkeit das zu meiner Arbeit erforderliche Germanium dedicirte, wurde es mir jetzt schon ermöglicht, festzustellen, mit welchem Elemente die Halogenübertragung in der Kohlenstoffgruppe beginnt; ich erlaube mir deshalb, demselben an dieser Stelle meinen verbindlichsten Dank auszusprechen für die freundliche Unterstützung meiner Untersuchungen!

In der Borgruppe wurden das Yttrium und Lanthan und zum Theil die Chloride dieser Metalle auf Halogenübertragung geprüft. Bei der Anwendung dieser und der später zu erwähnenden Elemente und ihrer Verbindungen wurde meist an zwei auf einander folgenden Tagen Chlor in Benzol, das mit den Ueberträgern beschickt worden war, eingeleitet. Zur Beurtheilung der Uebertragungstähigkeit der in Frage stehenden Körper hielt ich es für erforderlich, nochmals einen Fundamentalversuch mit Benzol ohne Zusatz anzustellen:

In $50 \mathrm{Grm} . \mathrm{C}_{6} \mathrm{H}_{6}$ wurde bei gewöhnlicher Temperatur und bei Lichtabschluss 18 Stunden Chlor eingeleitet. Am ersten Tage betrug die Gewichtszunahme 9 Grm., nach dem zweiten Tage nur noch $4 \mathrm{Grm}$. Ein solcher Gewichtsverlust nach dem Chloriren am zweiten Tage wurde meist auch bei Nichtüberträgern constatirt, so dass er für diese ziemlich 
392 Willgerodt: Die Halogenüberträger in den

charakteristisch ist. Bei der Destillation des gechlorten Benzols ergaben sich die folgenden Zahlen:

$$
\begin{aligned}
& \text { 1. } \quad 20^{\circ}-80^{\circ}=17 \text { Grm. } \\
& \text { 2. } 80^{\circ}-85^{\circ}=10 \quad ", \quad=27 \text { Grm. } \\
& \text { 3. } 85^{\circ}-100^{\circ}=6 \quad " \\
& \text { 4. } 100^{\circ}-150^{\circ}=2 \quad "
\end{aligned}
$$

Yttrium: 1. Versuch: 50 Grm. $\mathrm{C}_{6} \mathrm{H}_{6}+7 / 100$ Grm. Yttriummetall wurden 7 Stunden chlorirt. Gewichtszunahme 11 Grm. Destillation: 1. $20^{\circ}-82^{\circ}=26$ Grm., 2. $82^{\circ}-90^{\circ}$ $=15$ Grm.

2. Versuch: 50 Grm. $\mathrm{C}_{6} \mathrm{H}_{6}+{ }^{7} / 100$ Grm. $\mathrm{YCl}_{3}+6 \mathrm{H}_{2} \mathrm{O}$ wurden $71 / 4$ Stunden chlorirt. Gewichtszunahme $10 \mathrm{Grm}$. Destillation: $1.20^{\circ}-82^{\circ}=41 \mathrm{Grm}$., 2. $82^{\circ}-90^{\circ}=2 \mathrm{Grm}$.

3. Versuch: $50 \mathrm{Grm} . \mathrm{C}_{6} \mathrm{H}_{6}+{ }^{2} / 10$ Grm. $\mathrm{YCl}_{3}$, wasserfrei, wurden 15 Stunden chlorirt. Gewichtsverlust nach dem zweiten Tage 4 Grm. Gewichtszunahme 11 Grm. Destillation: $1.30^{\circ}-82^{\circ}=8 \mathrm{Grm} ., 2.82^{0}-85^{0}=5 \mathrm{Grm} ., 3.85^{0}-$ $95^{\circ}=18$ Grm., $4.90^{\circ}-130^{\circ}=2$ Grm., 5. $130^{\circ}-140^{\circ}$ : einige Tropfen.

Diese Versuche lehren, dass das Yttriumchlorid kein Halogenüberträger ist.

Lanthan: 50 Grm. $\mathrm{C}_{6} \mathrm{H}_{6}+0,1$ La wurden 9 Stunden chlorirt; das Metall wird scheinbar nur äusserst wenig vom Chlor angegriffen. Gewichtszunahme $10 \mathrm{Grm}$. Destillation: 1. bis $85^{\circ}=8$. Grm., 2. $85^{\circ}-100^{\circ}=21$ Grm., 3. $100^{\circ}-$ $130^{\circ}=10$ Grm., $130^{\circ}-210^{\circ}=8$ Grm.

Vergleicht man diese Zahlen mit denen des Fundamentalversuches, so muss man das metallische Lanthan als einen geringen Halogenüberträger ansprechen. Ein Versuch mit dem wasserfreien Chlorid soll noch ausgeführt werden.

Von den Halogenüberträgern der Elemente der $\mathrm{K}$ o hlenstoffgruppe war bis jetzt nur das Sn erkannt, ausserdem ist constatirt worden, dass die Elemente $\mathrm{C}, \mathrm{Si}$ und $\mathrm{Ti}$ nicht zu übertragen vermögen. Um das erste halogenübertragende Glied dieser Reihe aufzufinden, begann ich meine Versuche nochmals mit dem Titan und dehnte sie dann auf die folgenden, noch nicht untersuchten Elemente aus. 
matürlichen Gruppen u. d. Perioden der Elemente. 393

Versuch: 50 Grm. $\mathrm{C}_{6} \mathrm{H}_{6}+0,21$ Grm. Ti wurden 11 Stunden chlorirt. Gewichtsverlust am zweiten Tage 12 Grm. Gewichtszunahme $8 \mathrm{Grm}$. Destillation: $1.20^{\circ}-82^{\circ}=21 \mathrm{Grm}$., 2. $82^{\circ}-90^{\circ}=13$ Grm., 3. $90^{\circ}-150^{\circ}=5$ Grm.

Germanium: 1. Versuch: $50 \mathrm{Grm} . \mathrm{C}_{6} \mathrm{H}_{6}$ wurden in Gegenwart von $1 / 10$ Grm. Germanium 7 Stunden lang chlorirt und darauf während der Dauer einer Nacht stehen gelassen. Das Germanium wurde von dem Chlor durchaus nicht angegriffen und behielt Farbe, Glanz, Form und Gewicht vollständig bei, eine Chlorübertragung auf das Benzol konnte aus diesem Grunde nicht statthaben.

2. Versuch: 50 Grm. $\mathrm{C}_{6} \mathbf{H}_{6}+\mathrm{GeCl}_{4}(0,1$ Grm. Ge wurden durch Anwärmen im Chlorstrome verbrannt) wurden 10 Stunden mit Chlor behandelt. Gewichtsabnahme am zweiten Tage $3^{1 / 2}$ Grm. Gewichtszunahme $5^{1 / 2}$ Grm. Destillation: 1. $20^{\circ}-82^{\circ}=26 \mathrm{Grm} ., 2.82^{\circ}-90^{\circ}=9 \mathrm{Grm} ., 3.90^{\circ}-$ $150^{\circ}=2 \mathrm{Grm}$. Diese Zahlen beweisen, dass das Germanium nicht zu den Halogenüberträgern zählt.

Zirkonium: Durch einen einzigen Versuch mit dem metallischen Zirkonium konnte erwiesen werden. dass dieses Element ein ausgezeichneter und somit der erste Halogenüberträger dieser natürlichen Gruppe ist.

Versuch: 50 Grm. $\mathrm{C}_{6} \mathrm{H}_{6}+0,1$ Grm. $\mathrm{Zr}$ wurden 12 Stunden chlorirt. Gewichtszunahme $37 \mathrm{Grm}$. Destillation: 1. $130^{\circ}-140^{\circ}=7$ Grm., 2. $140^{\circ}-160^{\circ}=34^{1} / 2$ Grm., 3. $160^{\circ}-175^{\circ}=22$ Grm. (wird fest), 4. $175^{\circ}-215^{\circ}=7 \mathrm{Grm}$. (wird ebenfalls zum grossen Theil fest.)

Cer: 1. Versuch: 50 Grm. $\mathrm{C}_{6} \mathrm{H}_{6}+\% / 100$ Grm. Ce wurden $16^{1} /{ }_{4}$ Stunden bei $-5^{0}$ chlorirt. Gewichtsverlust am zweiten Tage 4 Grm. Gewichtszunahme 17 Grm. Destillation: 1. bis $82^{\circ}=18$ Grm., 2. $82^{\circ}-90^{\circ}=20$ Grm., 3. $90^{\circ}-130^{\circ}=7$ Grm., 4. $130^{\circ}-210^{\circ}=5$ Grm.

2. Versuch: $50 \mathrm{Grm} . \mathrm{C}_{6} \mathrm{H}_{6}+1 / 2 \mathrm{Grm} . \mathrm{Ce}_{2} \mathrm{Cl}_{6}+15 \mathrm{H}_{2} \mathrm{O}$ wurden $11^{1 / 2}$ Stunden chlorirt. Gewichtszunahme $12 \mathrm{Grm}$. Destillation: 1. bis $85^{\circ}=28$ Grm., 2. $85^{\circ}-100^{\circ}=11$ Grm. 3. $100^{\circ}-130^{\circ}=4 \mathrm{Grm}$.

3. Versuch: $50 \mathrm{Grm} . \mathrm{C}_{6} \mathrm{H}_{6}+0,5 \mathrm{Grm}$. eines weissen, 
394 Willgerodt: Die Halogenüberträger in den

wasserfreien Cerchlorides wurden 8 Stunden chlorirt. Gewichtszunahme $17 \mathrm{Grm}$. Beim Entfernen des gelösten Chlors mit Kalilauge wurde ein auf derselben schwimmendes Oel erhalten, das abgetrennt und über Chlorcalcium getrocknet wurde. Destillation des $45 \mathrm{Grm}$. wiegenden Oeles: 1. bis $82^{\circ}=29$ Grm., 2. $82^{\circ}-85^{\circ}=6$ Grm., 3. $85^{\circ}-110^{\circ}=2$ Grm. Diese Zahlen beweisen, dass die bis jetzt gewählten Verbindungsformen des Cers nicht zu übertragen vermögen.

Thorium: 1. Versuch: 50 Grm. $\mathrm{C}_{6} \mathrm{H}_{6}+0,1$ Grm. Th wurden 14 Stunden chlorirt; das Metall wurde dabei nur sehr wenig angegriffen. Die Destillation wurde erst nach 8 Tagen ausgeführt, sie ergab: 1 . bis $85^{\circ}=5 \mathrm{Grm}$., 2. $85^{\circ}-100^{\circ}=14 \mathrm{Grm}$. . 3. $100^{\circ}-130^{\circ} \mathrm{Grm} .=12$ Grm., 4. $130^{\circ}-170^{\circ}=5$ Grm.

2. Versuch: 50 Grm. $\mathrm{C}_{6} \mathrm{H}_{6}+0,1 \mathrm{Grm}$. ThCl $\mathrm{Th}_{4}$, wasserfrei, wurden 15 Stunden mit Chlor behandelt. Gewichtsabnahme nach Chlorirung des zweiten Tages war $9 \mathrm{Grm}$. Gewichtszunahme $11 \mathrm{Grm}$. Destillation: 1 . bis $85^{\circ}=13 \mathrm{Grm}$., 2. $85^{\circ}-100^{\circ}=22$ Grm., 3. $100^{\circ}-130^{\circ}=7$ Grm., 4. $130^{\circ}-$ $210^{\circ}=5$ Grm.

3. Versuch: Fast dieselben Zahlen wurden erhalten, als Th im Chlorstrome verbrannt und das erhaltene Produkt. zur Chlorübertragung benutzt wurde. Es muss somit das Thorium noch als ein äusserst schwacher Ueberträger angesprochen werden.

Die Elemente der Stickstoffgruppe sind als Halogenüberträger wieder leistungsfähiger, als die der vorhergehenden Reihe, denn sie weist als solche Vanadin, Niob, Antimon, Tantal und Wismuth auf.

Vanadin: Vexsuch: 50 Grm. $\mathrm{C}_{6} \mathrm{H}_{6}+1 / 2$ Grm. $\mathrm{Vd}_{2} \mathrm{O}_{2} \mathrm{Cl}_{1}+5 \mathrm{H}_{2} \mathrm{O}$ (+Vanadinoxyde) wurden ca. 14 Stunden chlorirt. Gewichtsabnahme nach dem zweiten Tage 3 Grm., Gewichtszunahme $8 \mathrm{Grm}$. Destillation: 1. bis $85^{\circ}=8 \mathrm{Grm}$., 2. $85^{\circ}-100^{\circ}=17$ Grm., 3. $100^{\circ}-130^{\circ}=17$ Grm. 4. $130^{\circ}-$ $180^{\circ}=8 \mathrm{Grm}$. Es ist hier interessant hervorzuheben, dass bei der Destillation sämmtliche Uebergangsprodukte rothbraun erscheinen, was darauf hindeutet, dass sich sehr wahrscheinlich $\mathrm{VdCl}_{4}$ während der Chlorirung bildet. Die 
natürlichen Gruppen $n$. d. Perioden der Elemente. 395 erhaltenen Zahlen deuten auf Halogenübertragung hin, und das Resultat dürfte besser ausfallen, wenn man von vorn herein das wasserfreie dunkelbraune $\mathrm{VdCl}_{4}$ anwendet (es wird ein solcher Versuch von mir ausgeführt werden).

Niob: 1. Versuch: 50 Grm. $\mathrm{C}_{6} \mathrm{H}_{8}+7 /{ }_{100}$ Grm. $\mathrm{Nb}$ wurden gegen 16 Stunden chlorirt. Gewichtsabnahme am zweiten Tag $2 \mathrm{Grm}$. Gewichtszunahme $12 \mathrm{Grm}$. Destillation: 1. bis $82^{\circ}=9 \mathrm{Grm}$., 2. $82^{0}-90^{\circ}=16 \mathrm{Grm}$. ., 3. $90^{\circ}-$ $130^{\circ}=14$ Grm., 4. $130^{\circ}-210^{\circ}=6$ Grm.

2. Versuch: 50 Grm. $\mathrm{C}_{6} \mathbf{H}_{6}+1$ Grm. wasserfreies Niobchlorid wurden gegen 18 Stunden chlorirt. Gewichtszunahme $8 \mathrm{Grm}$. (schlechter Verschluss). Destillation: 1. bis $130^{\circ}=6^{1} / 2$ Grm., 2. $130^{\circ}-140^{\circ}=20^{1} / 2$ Grm., 3. $140^{\circ}-$ $170^{\circ}=15$ Grm., 4. $170^{\circ}-210^{\circ}=4$ Grm. Deuten schon die mit dem Element gewonnenen Zahlen auf Chlorübertragung, so wird dieselbe mit Sicherheit festgestellt bei Anwendung des Chlorides.

Didym: Der erste Versuch wurde mit wasserhaltigem Didymchlorid ausgeführt und lieferte ein sehr ungünstiges Resultat: nach 7 stïndigem Chloriren gingen bei der Destillation $41 \mathrm{Grm}$. bis zu $82^{\circ}$ über; $50 \mathrm{Grm}$. Benzol waren zur Verwendung gelangt.

2. Versuch: 50 Grm. $\mathrm{O}_{6} \mathrm{H}_{6}+1$ Grm. wasserfreies Didymchlorid, 131/2 Stunden chlorirt, zeigten am Ende des zweiten Tages eine Gesammtgewichtszunahme von 14 Grm., einen Gewichtsverlust von $3 \mathrm{Grm}$. Destillation: 1. bis $82^{\circ}=28$ Grm., 2. $82^{\circ}-85^{\circ}=9$ Grm., 3. 85 $5^{\circ}-100^{\circ}=2$ Grm., 4. $100^{\circ}-130^{\circ}$ nicht $1 \mathrm{Grm}$. Zufolge dieser Resultate muss das Didym zu den Nichtüberträgern gezählt werden.

Tantal: 1 . Versuch: 50 Grm. $\mathrm{C}_{6} \mathrm{H}_{6}+{ }^{7}{ }_{100}$ Grm. Ta wurden ungefähr 17 Stunden chlorirt. Gewichtszunahme 13 Grm. Destillation: 1. bis $85^{\circ}=4$ Grm., 2. $85^{\circ}-100^{\circ}$ $10 \mathrm{Grm} ., 3.100^{\circ}-130^{\circ}=19 \mathrm{Grm} ., 4.130^{\circ}-170^{\circ}=7 \mathrm{Grm}$, 5. $170^{\circ}-210^{\circ}=2$ Grm.

2. Versuch: $50 \mathrm{Grm} . \mathrm{C}_{6} \mathrm{H}_{6}+{ }^{1 / 3}$ ? Grm. wasserfreies Tantalchlorid wurden gegen 17 Stunden chlorirt. Gewichtszunahme 20 Grm. Destillation. 1. bis $100^{\circ}=3$ Grm., 
396 Willgerodt: Die Halogenüberträger in den

2. $100^{\circ}-130^{\circ}=23$ Grm., 3. $130^{\circ}-140^{\circ}=13$ Grm., 4. $140^{\circ}-170^{\circ}=7$ Grm., 5. $170^{\circ}-210^{\circ}=3$ Grm. Das Tantal zählt somit zu den Halogenüberträgern.

Dass das Wismuth ein Ueberträger ist, habe ich bereits veröffentlicht.

Die bekannten Elemente der Sauerstoffgruppe sind nun, nachdem ich noch mit dem Uran experimentirt habe, sämmtlich auf Uebestragungsfähigkeit geprüft worden. Es hat sich bei den hierauf bezüglichen Arbeiten ergeben, dass dieser sechsten natürlichen Gruppe drei ganz vorzüglich brauchbare Glieder angehören: Mo, Te und Ur.

1. Versuch. Um mit Sicherheit das erste übertragende Element dieser Reihe festzustellen, wurde nochmals mit wasserfreiem sublimirten Chromchlorid gearbeitet.

50 Grm. $\mathrm{C}_{6} \mathrm{H}_{6}+1$ Grm. $\mathrm{Cr}_{2} \mathrm{Cl}_{6}$ wurden 14 Stunden chlorirt. Gewichtsabnahme am zweiten Tage $1 \mathrm{Grm}$. Gewichtszunahme $18 \mathrm{Grm}$. Destillation: 1 . bis $82^{\circ}=17 \mathrm{Grm}$., 2. $82^{\circ}-85^{\circ}=12$ Grm., 3. $85^{\circ}-90^{\circ}=6$ Grm., 4. $90^{\circ}-130^{\circ}$. $=5 \mathrm{Grm}$. Hieraus ist zu ersehen, dass dieses Chlorid total unfähig ist, Chlor zu übertragen.

Nach dieser Einsichtnahme musste" um so mehr daran gezweifelt werden, dass der Schwefel das erste übertragende Glied der Sauerstoffgruppe ist, da derselbe bei gewöhnlicher Temperatur keine übertragende Verbindungsform zu bilden vermag: Versuche mit dem Schwefel oder seinen Verbindungen mussten wiederholt und der bestehende Widerspruch gehoben werden.

2. Versuch: 50 Grm. $\mathrm{C}_{6} \mathrm{H}_{6}+2$ Grm. $\mathrm{S}_{2} \mathrm{Cl}_{2}$ (chemisch rein erhalten durch mehrfache Destillation) wurden gegen 14 Stunden chlorirt. Gewichtsabnahme am zweiten Tage $4 \mathrm{Grm}$. Gesammtgewichtszunahme $10 \mathrm{Grm}$. Destillation: 1. $20^{\circ}-82^{\circ}=26$ Grm., 2. $82^{0}-85^{\circ}=13$ Grm., 3. $85^{\circ}-$ $100^{\circ}=5$ Grm. , 4. $100^{\circ}-130^{\circ}=2^{1 / 2}$ Grm. Nach diesen Thatsachen kann es keinem Zweifel unterliegen, dass der Schwefel kein Halogenüberträger ist, und dass der früher von mir mit dem Schwefel erzielte Erfolg einer Verunreinigung der angewandten Schwefelblumen zugeschrieben werden muss. 
natürlichen Gruppen u. d. Perioden der Elemente. 397

Die Resultate, die von anderer, sowie auch von meiner Seite mit dem Mo, Te und $\mathrm{W}$ gewonnen wurden, sind veröffentlicht worden, es konnte sich also nur noch um das Uran handeln.

3a. Versuch: $50 \mathrm{Grm} . \mathrm{C}_{6} \mathrm{H}_{6}+{ }^{1 / 10}$ Grm. Ur wurden gegen 14 Stunden chlorirt. Gewichtszunahme 7 Grm. Destillation: 1. bis $85^{\circ}=5$ Grm., 2. $85^{\circ}-100^{\circ}=15 \mathrm{Grm} ., 3.100^{\circ}-$ $130^{\circ}=18$ Grm., 4. $130^{\circ}-210^{\circ}=10$ Grm.

$3 \mathrm{~b}$. Versuch: $50 \mathrm{Grm} . \mathrm{O}_{6} \mathrm{H}_{6}+1 / 2$ Grm. Uranylchlorid, wasserhaltig, wurden 7 Stunden chlorirt, aber durchaus keine Einwirkung erzielt: die Gewichtszunahme betrug nur 5 Grm.

3c. Versuch: 50 Grm. $\mathrm{C}_{5} \mathrm{H}_{6}+1 \mathrm{Grm}$. $\mathrm{UCl}_{4}$, wasserfrei, wurden an einem Tage $9^{1} / 2$ Stunden chlorirt. Das Uranchlorür löst sich in dem Benzol mit dunkelrothbrauner Farbe und überträgt das eintretende Chlor in so vorzüglicher Weise, dass sich in der Aufsatzröhre kein Benzolhexachlorid bildet. Die nachfolgenden Zahlen zeigen, dass die angewandte Verbindung hinsichtlich der Uebertragung den Eisenverbindungen an die Seite zu stellen ist. Die Gewichtszunahme betrug $30 \mathrm{Grm}$. und die Destillation ergab: 1. Von $130^{\circ}-140^{\circ}=2^{1} / 2$ Grm., 2. $140^{\circ}-160^{\circ}=26$ Grm., 3. $160^{\circ}-180^{\circ}=28 \mathrm{Grm}$; diese Fraktion wurde zum grössten Theile fest; dass $p$-Dichlorbenzol vorhanden war, zeigte sich schon vor der Destillation, da dasselbe aus der flüssigen Masse auskrystallirte.

In der Fluorgruppe haben wir bis jetzt nur einen einzigen und zwar den längst bekannten und vielfach angewandten Ueberträger, das Jod.

Nach den von mir aufgefundenen Thatsachen ist es jedoch nun höchst wahrscheinlich, dass das noch unbekannte „Ekamangan“, mit dem Atomgewicht 100, das übertragende Anfangsglied dieser Reihe sein wird.

Am Schlusse meiner Abhandlung angelangt, unterlasse ich es nicht, die Halogenüberträger nach dem periodischen System der Elemente zusammenzustellen: 
398 Willgerodt: Die Halogenüberträger in den

Tabelle der Halogenüberträger im periodischen System der Elemente')

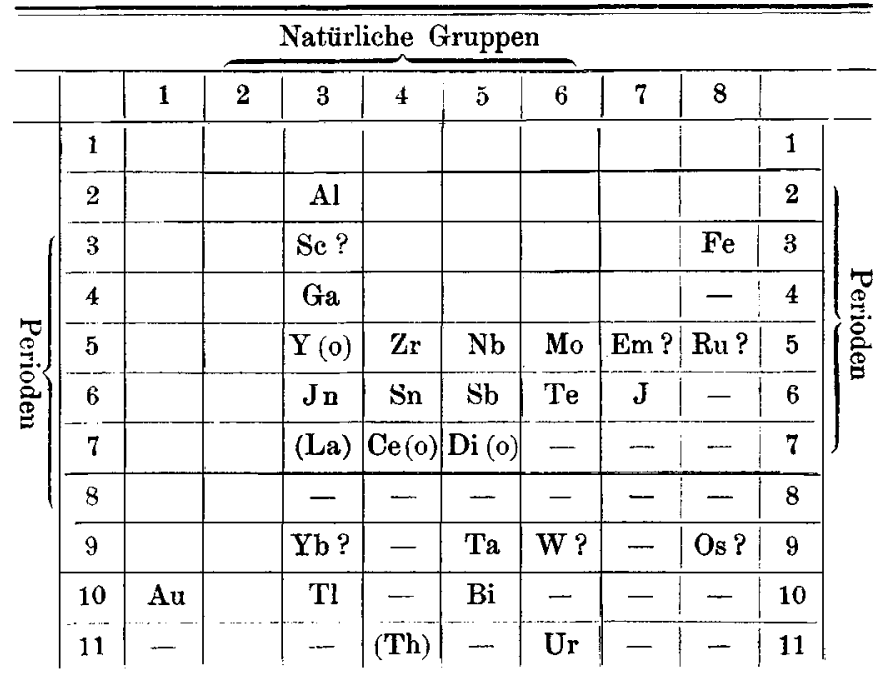

Es hat sich somit herausgestellt, duss sich die von mir aufgestellte Regel über den Zusammenhang der Halogenübertragungsfähigkeit der Elemente mit ihren Atomgewichten und Verbindungsformen im Grossen und Ganzen bewährt hat, dass dieselbe jedoch nicht ohne Ausnahme ist, wenn die dem $\mathrm{Y}, \mathrm{Ce}$ und $\mathrm{Di}$ zugeschriebenen Atomgewichte und die von diesen abhängigen Verbindungsformen bestätigt werden: denn diese drei Elemente vermögen durchaus kein Chlor auf Benzol zu übertragen. - Wie dem nun aber auch sei, meiner Meinung nach ist es nicht zu verkennen, dass das periodische System der Elemente einen neuen Sieg davon getragen hat: auf Grund desselben lassen sich die Halogenüberträger mit grosser Wahrscheinlichkeit voraus-

1) Die in dieser Tabelle eingeklammerten Elemente sind schwache, die mit $(0)$ bezeichneten, keine Ueberträger. Ein ? hinter dem Symbol soll andeuten, dass das betreffende Element noch gar nicht oder doch nicht genügend auf Halogenübertragung untersucht worden ist. Jeder Strich in einer Rubrik deutet ein noch fehlendes, auf Halogenübertragung zu untersuchendes Element an. 
sagen, und diese Wahrscheinlichkeit wird um so grösser werden, je mehr man die Factoren erkennen wird, die die in Rede stehende Eigenschaft bedingen; von diesen dürften ausser dem Atomgewicht und der Verbindungsform auch noch andere, so z. B. die Löslichkeit der übertragenden Form in der zu halogenirenden aromatischen Substanz eine nicht unwesentliche Rolle spielen. - Die Chloride des $\mathrm{Y}$, Ce und Di scheinen gänzlich unlöslich in Benzol zu sein, und sehr wahrscheinlich hängt auch die geringe Wirkung der wasserfreien Chloride des La und $\mathrm{Th}$, sowie des $\mathrm{Nb}$ und Ta von diesem Factor ab, denn es wurde bemerkt, dass das Benzol nur wenig von diesen eingetragenen Substanzen zu lösen vermochte.

Schliesslich sei noch darauf hingewiesen, dass überall da, wo Halogenverbindungen zu übertragen vermögen, auch die ihnen zu grundeliegenden Elemente als Ausgangsmaterialien verwendet werden können, dass dieselben aber in manchen Fällen weit hinter jenen Verbindungen in ihrer Wirkung zurückstehen, und dies ist selbstverständlich immer dann der Fall, wenn sich die Halogenverbindungen beim Zusammentreffen der Elemente in äusserst geringen Mengen bilden. Wenngleich die Chloride des Mo und Ur vorzügliche Ueberträger sind, so üben die Metalle selbst doch nur einen geringen Einfluss aus; ähnlich verhalten sich $\mathrm{Nb}$ und Ta und ihre Chlorverbindungen. Die Chloride von La und Th dagegen veranlassen einen ebenso geringen Erfolg als diese Metalle selbst.

Die Elemente, die als solche mehr oder weniger brauchbar für Halogenübertragung sind und verwendet werden können, sind: $\mathrm{Au}, \mathrm{Al}, \mathrm{Ga}, \mathrm{Jn}, \mathrm{Tl}, \mathrm{Zr}, \mathrm{Sn}, \mathrm{Sb}, \mathrm{Bi}, \mathrm{Te}, \mathrm{J}$ und Fe.

Oxyde und Hydroxyde, sowie auch Sulfide und Salze halogenübertragender Elemente versagen in manchen Fällen den Dienst für besagten $Z$ weck, hierher zählen z. B. Verbindungen des $\mathrm{Al}$ und des Mo.

Nach den bis jetzt gemachten Erfahrungen zeigen Antimon und Eisen insofern eine ganz ausserordentliche Uebertragungstendenz, als sich auch jene gedachten Verbindungen 
$400 \mathrm{Klason}$ : Ueb. d. freie Thiocyansäure u. Cyansäure

als vorzügliche Ueberträger herausgestellt haben. Vom Eisen wurde bislang nur eine einzige Ausnahmeverbindung aufgefunden: das Ferrocyankalium. Dieses Doppelcyanid vermochte kein Chlor substituirend in das Benzol einzuführen; es übte im zerstreuten Tageslichte, ähnlich wie der Alaun, - wenn auch nicht in so hervorragendem Maasse - einen addirenden Einfluss aus.

Hiermit beschliesse ich zunächst meine Arbeit über Halogenüberträger; der im System noch nicht berücksichtigten Elemente werde ich gedenken, sobald sich mir die Gelegenheit dazu bietet.

Freiburg i. B., im März 1887.

\section{Ueber die freie Thiocyansäure und Cyansãure und ihre Verbindungen mit Aether und Alkoholen; \\ Voll}

Peter Klason.

I. Die wasserfreie Rhodanwasserstoffsäure, ihre Darstellung und Eigenschaften.

Seine Versuche über die Darstellung von wasserfreier Rhodanwasserstoffsäure beschreibt $\mathrm{W} \ddot{\mathrm{hhle}} \mathrm{r}^{1}$ ) folgendermassen: „Ich brachte kleine, bei $80^{\circ}$ getrocknete Kugeln von Schwefelcyanquecksilber in Hydrothiongas, das über Quecksilber gesperrt war. So oft man den Versuch wiederholt, zeigen sich stets folgende Erscheinungen: Die Kugeln werden sogleich gelb und gleich darauf schwarz, das Quecksilber fängt an $\mathrm{zu}$ steigen und an der Wand läuft eine wasserhelle Flüssigkeit in ätherartigen Streifen herunter, die aber sehr bald gelb wird und dann zu einer pommeranzengelben Materie erstarrt. Dreht man die Röhre herum, so

1) Gilb. Ann. 1821, 272. 